\title{
Câncer de mama: percepções frente à mastectomia
}

\author{
Breast cancer: perceptions regarding mastectomy \\ Cáncer de mama: percepciones sobre la mastectomia
}

Recebido: 3011/2021 | Revisado: 04/11/2021 | Aceito: 04/12/2021 | Publicado: 05/12/2021

\author{
Rochely Souza Dias \\ ORCID: https://orcid.org/0000-0002-7177-114X \\ Centro Universitário FAMETRO, Brasil \\ E-mail: souzarochely@gmail.com \\ Elaine dos Santos Maia \\ ORCID: https://orcid.org/0000-0003-2473-4941 \\ Centro Universitário FAMETRO, Brasil \\ E-mail: elainemaia47@gmail.com \\ Graciana de Souza Lopes \\ ORCID: https://orcid.org/0000-0003-3615-9040 \\ Centro Universitário FAMETRO, Brasil \\ E-mail: gracilopess@hotmail.com
}

\begin{abstract}
Resumo
Introdução: $O$ câncer de mama se apresenta através do desenvolvimento desorganizado e desordenado das células, passam a ser incontroláveis e agressivas, contribuindo com a formação de tumores malignos. Objetivo: Discutir sobre as percepções de mulheres frente a mastectomia. Metodologia: Revisão de literatura, utilizando como fontes de pesquisa, as bases de dados Scientific Electronic Library On-line (SCIELO) e Literatura Latino Americana e do Caribe em Ciências da Saúde (LILACS), foram selecionados os artigos que entraram nos critérios de inclusão, que estavam nos períodos de publicação de 2012 a 2021 e que possuem relação direta com a temática. Resultado: Constatou-se que as mulheres mastectomizadas, por um lado, apresentam dificuldades físicas com a retirada da mama, o que afeta sua autoimagem, com sentimento de dor, tristeza e revolta pela mutilação. De outro, ainda demonstram sentimentos de esperança pelo apoio da família e da relação com outras mulheres que vivenciam a mesma experiência da mastectomia. Conclusão: O Enfermeiro pode contribuir através de condutas educativas, com a melhor qualidade de vida das pacientes mastectomizadas, assim como, para o alcance de um tratamento humanizado, é importante atuar no pré-operatório e reabilitação da mulher.
\end{abstract}

Palavras-chave: Câncer de mama; Mulher; Mastectomia; Enfermeiro.

\begin{abstract}
Introduction: Breast cancer presents itself through the disorganized and disordered development of cells, becoming uncontrollable and aggressive, contributing to the formation of malignant tumors. Objective: Discuss the perceptions of women facing mastectomy. Methodology: Literature review, using as research sources the Scientific Electronic Library On-line (SCIELO) and Latin American and Caribbean Literature in Health Sciences (LILACS) databases, the articles that met the inclusion criteria were selected., which were in the publication periods from 2012 to 2021 and which have a direct relationship with the theme. Results: It was found that women with mastectomies, on the one hand, have physical difficulties with the removal of the breast, which affects their self-image, with feelings of pain, sadness and anger due to the mutilation. On the other hand, they still show feelings of hope for the support of the family and the relationship with other women who experience the same experience of mastectomy. Conclusion: Nurses can contribute through educational approaches, with a better quality of life for mastectomized patients, as well as, for the achievement of a humanized treatment, it is important to act in the preoperative and rehabilitation of women.
\end{abstract}

Keywords: Breast cancer; Woman; Mastectomy; Nurse.

\section{Resumen}

Introducción: El cáncer de mama se presenta a través del desarrollo desorganizado y desordenado de las células, volviéndose incontrolable y agresivo, contribuyendo a la formación de tumores malignos. Objetivo: discutir las percepciones de las mujeres que se enfrentan a una mastectomía. Metodología: Revisión de la literatura, utilizando como fuentes de investigación las bases de datos de la Biblioteca Científica Electrónica en Línea (SCIELO) y Literatura Latinoamericana y del Caribe en Ciencias de la Salud (LILACS), se seleccionaron los artículos que cumplieron con los criterios de inclusión, que se encontraban en los períodos de publicación. de 2012 a 2021 y que 
tienen una relación directa con la temática. Resultados: Se encontró que las mujeres con mastectomías, por un lado, tienen dificultades físicas con la extracción de la mama, lo que afecta su autoimagen, con sensaciones de dolor, tristeza e ira por la mutilación. Por otro lado, aún demuestran sentimientos de esperanza por el apoyo de la familia y la relación con otras mujeres que experimentan la misma experiencia de mastectomía. Conclusión: Las enfermeras pueden contribuir a través de enfoques educativos, con una mejor calidad de vida para las pacientes mastectomizadas, así como, para la consecución de un tratamiento humanizado, es importante actuar en el preoperatorio y rehabilitación de la mujer.

Palabras clave: Cáncer de mama; Mujer; Mastectomía; Enfermero.

\section{Introdução}

O câncer é um termo genérico utilizado para identificar o grupo com mais de 100 doenças, que possuem o crescimento desequilibrado de células que comprometem os órgãos e os tecidos, e podem se expandir para outros locais do corpo. Ao se dividirem rapidamente, estas células podem se tornar bastante agressivas e irrefreáveis, resultando no desenvolvimento de tumores, identificadas como células cancerosas, entre eles, está o câncer de mama (Batista, 2015).

O câncer de mama se define como uma neoplasia que envolve uma das mamas e por vezes as axilas, apresentando-se como um nódulo resistente que pode ser indolor ou não, ainda pode se manifestar através das alterações na pele, secreção mamilar, ou intensa dor na mama (Alves, 2016). É o que afirmam, Ferlay, et al., (2019) o câncer de mama é classificado como uma neoplasia maligna mais recorrente em mulheres.

Se apresenta como um grave problema de saúde pública devido ao aumento gradativo de casos recorrentes no decorrer do tempo, segundo estimativas da Organização Mundial de Saúde (OMS) serão em 2030, diagnosticados 22 milhões em todo o mundo, novos casos da doença (Inca, 2019).

A prevenção secundária se baseia no Exame Clínico das Mamas (ECM) desenvolvido por enfermeiros ou médicos treinados e também no rastreamento realizado através da mamografia. Anualmente, todas as mulheres a partir dos 40 anos, devem se sujeitar ao exame clínico das mamas e as mulheres classificadas como grupo de risco superior, devem realizar o ECM exame anual, a partir dos 35 anos (Ohll, et al., 2016).

É importante enfatizar que se comparado a outras patologias, o câncer de mama possui controle com especificidades, são métodos que iniciam da prevenção primária, subtração de amostras a componentes cancerígenos, e também da compreensão que a mulher possui sobre a doença. A melhor probabilidade de cura e de tratamento ocorre a partir do melhor autoconhecimento sobre a neoplasia em relação ao corpo (Silva, et al., 2015).

Nas mulheres são geradas angústias, a partir da apreensão da doença até o diagnóstico, devido às reações do tratamento e da concepção de óbito relacionado ao câncer. O tratamento do câncer pode envolver mais de um método terapêutico, como radioterapia, quimioterapia, terapia alvo molecular e cirurgia (mastectomia) (Medeiros, et al., 2019).

Na percepção após a mastectomia, se considera que a mutilação atinge a autopercepção da paciente, resultando em constrangimento, fragilidade por cicatrizes derivadas do método cirúrgico, identificando baques sexuais. Na apresentação da recorrência, destaca-se o pensamento do diagnóstico com uma contínua na vida das mulheres, o que gera sentimento de ansiedade, angústia, medo e depressão (Vale, et al., 2017).

Pesquisas analisam o efeito do diagnóstico e tratamento, posto que, a mulher experimenta a compreensão de um futuro duvidoso, de um percurso desconhecido com grandes desafios, devido ao receio de mutilação ou morte. Frente deste fato desvelado, compreende-se que o Enfermeiro pode auxiliar positivamente na promoção de saúde das mulheres mastectomizadas, se apresentando como um componente essencial no processo de cuidado.

A justificativa deste estudo relaciona-se ao processo de enfrentamento do câncer de mama feminina, considerando a interface e similaridade entre muitos processos psicológicos, vivenciados após o diagnóstico que repercutem negativamente, tanto no corpo, quanto no psiquismo da mulher. Este estudo tem relevância social, pois, pode contribuir para elevar o 
conhecimento das mulheres sobre os autoexames de rotina da mama, visando reduzir o câncer de mama na população feminina, por ainda se apresentar como grave problema de saúde pública.

Em relação à relevância profissional, é importante que o profissional de enfermagem tenha melhor compreensão de como ele pode atuar no combate, prevenção ao câncer de mama, na orientação e apoio emocional as mulheres mastectomizadas, pois, contribui diretamente na administração, reabilitação das pacientes, oferecendo-lhes apoio emocional.

Deste modo, este estudo tem como objetivo discutir sobre as percepções de mulheres frente a mastectomia.

\section{Metodologia}

Para coleta de dados, foram utilizadas as bibliotecas virtuais de pesquisa: Biblioteca Científica Eletrônica Online (SCIELO) e Literatura Latino Americana e do Caribe em Ciências da Saúde (LILACS) mediantes os seguintes descritores: "câncer de mama", "mastectomia" e "enfermagem".

Como critérios de elegibilidade foram: selecionados artigos originais, disponibilizados gratuitamente, em língua portuguesa e língua inglesa, publicados no período de 2012 a 2020, que tratam do tema pesquisado.

Critérios de inelegibilidade foram: artigos com texto incompleto, resumos, monografias, dissertações de mestrado e teses de doutorado.

Os artigos foram selecionados conforme os critérios de elegibilidade e inelegibilidade a partir de seus títulos, posteriormente, foi realizada a análise de resumos e finalmente os artigos foram lidos na íntegra, elaborado um instrumento para a coleta de informações direto das bases de dados (Figura 1).

Figura 1: Seleção dos artigos para a revisão de literatura.

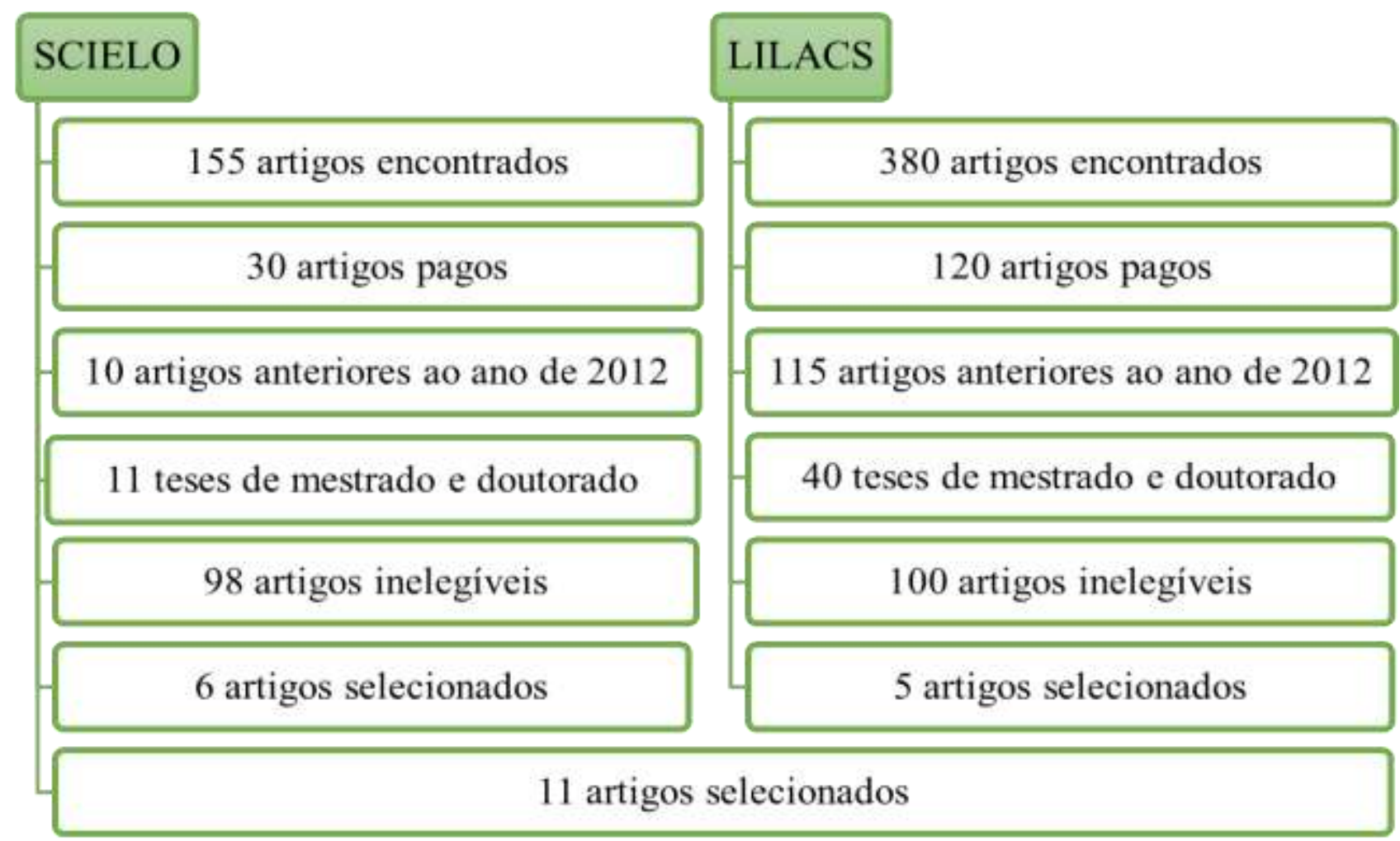

Fonte: Rochely, et al. (2021). 


\section{Resultados}

As pesquisas nas bases de dados (SCIELO e LILACS), com a utilização dos descritores em saúde nos proporcionaram um total de 535 artigos que teriam possibilidades para o uso da formulação da revisão desejada, depois de realizadas as leituras dos mesmos, e avaliando quanto à relevância e à importância de cada artigo e aplicando os critérios de elegibilidade e inelegibilidade, foram selecionados 20 trabalhos que respondiam aos objetivos propostos para a revisão. Dessa forma, após serem relidos e reavaliados, ficaram 11 estudos que chegaram ao objetivo desta revisão de literatura.

Os artigos selecionados para a revisão foram sujeitos a um estudo criterioso e leitura minuciosa. Sendo trabalhadas as informações extraídas das pesquisas selecionadas, estruturadas e sumarizadas de forma concisa, formando um banco de dados simples, de fácil compreensão e manejo. (Tabela 1).

Tabela 1: Resumo dos resultados dos estudos selecionados.

\begin{tabular}{|c|c|c|c|c|c|}
\hline & TÍTULO & ANO/ PAÍS & AUTORES & $\begin{array}{l}\text { BASE DE } \\
\text { DADOS }\end{array}$ & RESULTADOS \\
\hline 1 & $\begin{array}{l}\text { As Transformações } \\
\text { biopsicossociais em mulheres } \\
\text { mastectomizadas. }\end{array}$ & $\begin{array}{l}2016 \\
\text { Brasil }\end{array}$ & $\begin{array}{l}\text { Azevedo, et al., } \\
2016\end{array}$ & Scielo & $\begin{array}{l}\text { As mulheres mastectomiza-das apresentam sentimentos } \\
\text { de negatividade ligados ao temor do procedimento e da } \\
\text { morte, da falta de autoestima que acaba levando ao } \\
\text { preconceito social e a experiência da sexualidade. }\end{array}$ \\
\hline 2 & $\begin{array}{l}\text { Sexualidade de câncer de } \\
\text { mama. }\end{array}$ & $\begin{array}{l}2018 \\
\text { Brasil }\end{array}$ & $\begin{array}{l}\text { Toilier, et al., } \\
2018\end{array}$ & Scielo & $\begin{array}{l}\text { Cada mulher experimentou vivencias do câncer de } \\
\text { mama de maneira de forma peculiar, todas manifestaram } \\
\text { sentimentos psicológicos e físicos. }\end{array}$ \\
\hline 3 & $\begin{array}{l}\text { Qualidade de vida em } \\
\text { mulheres com câncer de } \\
\text { mama pós-intervenção } \\
\text { cirúrgica em uma cidade da } \\
\text { zona da mata de Minas } \\
\text { Gerais, Brasil. }\end{array}$ & $\begin{array}{l}2017 \\
\text { Brasil }\end{array}$ & $\begin{array}{l}\text { Barbosa, et } \\
\text { al., } 2017\end{array}$ & Scielo & $\begin{array}{l}\text { As mulheres que realizaram radioterapia alcançaram } \\
\text { boas percepções, principalmente as que tiveram os } \\
\text { linfonodos ressecados. }\end{array}$ \\
\hline 4 & $\begin{array}{l}\text { Construção de instrumentos } \\
\text { para o cuidado sistematizado } \\
\text { da enfermagem: mulheres em } \\
\text { processo cirúrgico de } \\
\text { mastectomia. }\end{array}$ & $\begin{array}{l}2016 \\
\text { Brasil }\end{array}$ & $\begin{array}{l}\text { Paiva, et al., } \\
2016\end{array}$ & Lilacs & $\begin{array}{l}\text { É importante que o trabalho do enfermeiro seja baseado } \\
\text { em uma própria compreensão científica e num contexto } \\
\text { interdisciplinar, o que contribui diretamente com a } \\
\text { percepção do enfermeiro como profissional emancipado. }\end{array}$ \\
\hline 5 & $\begin{array}{l}\text { Prevalência de Ansiedade e } \\
\text { Depressão em Pacientes } \\
\text { Oncológicos e Identificação } \\
\text { de Variáveis Predisponentes. }\end{array}$ & $\begin{array}{l}2016 \\
\text { Brasil }\end{array}$ & $\begin{array}{l}\text { Ferreira, et } \\
\text { al., } 2016\end{array}$ & Lilacs & $\begin{array}{l}\text { Pacientes oncológicos geralmente são acometidas por } \\
\text { distúrbios prevalentes como ansiedade e depressão. }\end{array}$ \\
\hline 6 & $\begin{array}{l}\text { Câncer de mama e reação } \\
\text { emocionais: Revisão } \\
\text { Sistemática. }\end{array}$ & $\begin{array}{l}2016 \\
\text { Brasil }\end{array}$ & $\begin{array}{l}\text { Arab, et al., } \\
\quad 2016\end{array}$ & Lilacs & $\begin{array}{l}\text { Em todos os processos do câncer de mama, as mulheres } \\
\text { demonstraram alterações emocionais de medo, surpresa, } \\
\text { ansiedade e alterações com imagem corporal. }\end{array}$ \\
\hline 7 & $\begin{array}{l}\text { Câncer de mama: } \\
\text { sentimentos e percepções das } \\
\text { mulheres diante do } \\
\text { diagnóstico. }\end{array}$ & $\begin{array}{c}2018 \\
\text { Brasil }\end{array}$ & $\begin{array}{l}\text { Mattias, et al., } \\
\quad 2018\end{array}$ & Lilacs & $\begin{array}{l}\text { A pesquisa demonstrou que foi possível compreender } \\
\text { percepções e sentimentos de mulheres diante a } \\
\text { investigação do câncer de mama. }\end{array}$ \\
\hline 8 & $\begin{array}{l}\text { Experiência em centro de } \\
\text { reabilitação de mulheres } \\
\text { mastectomizadas no início da } \\
\text { pandemia do COVID-19. }\end{array}$ & $\begin{array}{l}2021 \\
\text { Cuba }\end{array}$ & $\begin{array}{c}\text { Tapia, et al., } \\
2021\end{array}$ & Scielo & $\begin{array}{l}\text { O cuidado realizado por uma equipe interdisciplinar se } \\
\text { torna essencial na atenção as mulheres mastectomizadas, } \\
\text { posto que, a neoplasia geram inúmeras transformaçoes } \\
\text { na vida das mulheres. }\end{array}$ \\
\hline 9 & $\begin{array}{l}\text { Qualidade de vida em } \\
\text { mulheres com neoplasias de } \\
\text { mama em quimioterapia. }\end{array}$ & $\begin{array}{l}2014 \\
\text { Brasil }\end{array}$ & $\begin{array}{l}\text { Lôbo, et al., } \\
\qquad 2014\end{array}$ & Scielo & $\begin{array}{l}\text { As mulheres com câncer de mama demonstraram } \\
\text { modificações na satisfação pessoal, no comando } \\
\text { emocional e financeiro e nas futuras expectativas. }\end{array}$ \\
\hline 10 & $\begin{array}{l}\text { Ações/plano de alta da } \\
\text { enfermagem à mulher } \\
\text { submetida à mastectomia. }\end{array}$ & $\begin{array}{l}2020 \\
\text { Brasil }\end{array}$ & $\begin{array}{c}\text { Scofano, et al., } \\
2020\end{array}$ & Scielo & $\begin{array}{l}\text { Grande parte dos artigos destacou que as ações do } \\
\text { enfermeiro nos planos de alta a mulheres sujeitas à } \\
\text { mastectomia sendo fundamentada na educação constante } \\
\text { das mulheres e de seus familiares. }\end{array}$ \\
\hline 11 & $\begin{array}{l}\text { A importância da } \\
\text { enfermagem no pós- } \\
\text { operatório de mulheres } \\
\text { mastectomizadas com } \\
\text { dissecção de linfonodos } \\
\text { axilares: revisão integrativa. }\end{array}$ & $\begin{array}{l}2021 \\
\text { Brasil }\end{array}$ & $\begin{array}{l}\text { Silva, et al., } \\
2021\end{array}$ & Lilacs & $\begin{array}{l}\text { A enfermagem nas orientações de mulheres com } \\
\text { insuficiência de aprendizagem voltadas ao acolhimento } \\
\text { as mulheres e mastectomia de mulheres com transtornos } \\
\text { de imagem corporal. Constatou-se que as atenções } \\
\text { realizadas pelo enfermeiro em mulheres durante a } \\
\text { recuperação pós- mastectomia. }\end{array}$ \\
\hline
\end{tabular}

Fonte: Rochely, et al. (2021). 


\section{Discussão}

\subsection{Características e sintomas do câncer de mama benigno e maligno}

Atualmente, o câncer de mama é o mais recorrente na população feminina brasileira (com ressalva do câncer de pele não melanoma). De acordo com dados do Instituto Nacional de Câncer (Inca, 2019), somente em 2020, ocorreram 66.280 casos novos de câncer de mama, representando 29,7\% das ocorrências em mulheres (Teixeira, et al., 2020).

Mesmo diante de vários anos de diligências políticas públicas e médicas, as taxas de mortalidade da doença permanecem altas no Brasil. Estudos apontam que esses índices se mantêm elevados, porque a patologia ainda é diagnosticada em períodos avançados. No Brasil, o câncer de mama, é definido como um problema de saúde pública. Deste modo, o sistema de saúde passa por um grande desafio, como assegurar o acesso total e equilibrado das mulheres no que se refere ao diagnóstico e tratamento (Zapponi, et al., 2015).

Segundo Teixeira e Neto (2020), as direções que levaram o CM a situação de grande problema de Saúde Pública, a ser um tema constante de debates no Brasil, por causar a morte em muitas mulheres, ademais continua crescendo. No século XX, aconteceram mudanças radicais nas formas de entender, abordar e representar o câncer. Problemas inespecíficos, sem cura e de baixa incidência, o câncer se tornou um mal cada vez mais presente, conhecido e temido de modo geral na sociedade brasileira, assim, como mundialmente.

Atualmente, o CM é o mais ativo na população feminina no Brasil (com exceção do câncer de pele não melanoma). Conforme afirmações do Instituto Nacional de Câncer (INCA), em 2020, deverão ocorrer 66.280 novos casos de CM, estimando 29,7\% dos casos na população feminina. Embora décadas de trabalhos médicos e políticas públicas, as taxas de mortalidade da patologia continuam altas na sociedade brasileira (Inca, 2019).

Diversas transformações permitem que uma célula somente se estruture, porém, não possam afetar outros tecidos, denominados, tumores não cancerosos ou benignos. Para que uma célula seja determinada cancerígena, é relevante ocorrer modificações no material genético de uma ou diversas células, para estas poderem se dividir, evitando a morte celular (Santos, et al., 2018).

Entre os sintomas existentes, estão as mudanças físicas nas mamas que podem ser indícios de CM. Para prevenção do CM é fundamental que todas as mulheres façam o exame físico, pois, quando a mulher conhece o próprio corpo, pode ser possível observar sintomas e sinais iniciais, como: pele da mama retraída ou avermelhada, caroço fixo, indolor e normalmente endurecido, mudanças no bico do peito, pequenos nódulos nas regiões do pescoço, embaixo dos braços e saída espontânea de líquido dos mamilos (Inca, 2017).

Os sintomas e sinais do câncer de mama são: modificações na forma ou tamanho da mama, inchaço, vermelhidão, caroço ou nódulo na mama não reduz e se encontra sempre presente, dor na pele ou calor na mama, nódulos e inchaços contínuos nas ínguas das axilas, existe assimetria entre às duas mamas, ou seja, uma tem maior proporção do que a outra, enrijecimento da pele da mama, existência de sulco na mama, coceira recorrente no mamilo ou na mama, feridas na pelo e formação de crostas (Santos, et al., 2018).

Ao explanar sobre as neoplasias benignas, pode-se constatar que exibem aumento lento de propagação continua não afetando outro tecido, porém, elas reprimem os tecidos próximos, resultando na redução do parênquima e da atrofia, sendo assim, é suprimido o tecido sem modificações, predominando estroma reduzido, sustentando uma superfície de tecido conjuntivo fibroso que envolve a parti de uma cápsula o câncer (Costa, 2019).

Grande parte dos tumores malignos de mama são definidos como carcinoma ductal infiltrante. Essa categoria de câncer de mama desenvolve nos canais de leite. O câncer de mama ainda se apresenta principalmente em mulheres, porém pode- se apresentar em homens, apesar de ser raro. Considera-se que o período de 6 a 8 anos para que o gânglio afete a mama, 1 centímetro de diâmetro (Borba, 2018). 


\subsection{Aspectos Relacionados a Mastectomia}

No estudo de Ferreira, et al. (2016) onde sua pesquisa apresenta que 233 pacientes, sendo 65\% mulheres; 55\% entrevistados no setor de quimioterapia; e 37\% com até três anos de tratamento. Entre os entrevistados, foram encontrados $31,33 \%$ com ansiedade prováveis ou possíveis, e 26,18\% depressivos prováveis ou possíveis. Nos trabalhos de Arab, et al. (2016), os resultados do estudo descrevem que em todas as etapas do câncer de mama, as mulheres do Brasil apresentam reações emocionais de surpresa, medo, indignação, ansiedade, distúrbio com imagem corporal, que podem estar relacionadas à relação médico-paciente.

Para Guedes, et al. (2018), um dos procedimentos de tratamento do CM é a mastectomia, pelo qual, está na retirada total da mama, com ou sem exclusão de linfonodos axilares. Logo, pode ser indicado dependentemente da proporção do volume tumoral relacionado ao tamanho da mama. Segue como uma competência durante o recurso terapêutico como eficaz, porém mutilador, em que retira uma glândula da mulher com elevado sinal estético e sexual.

Segundo Pereira, et al. (2017), a mastectomia tem uma característica dura e traumática para a vida da mulher com CM por ser um procedimento cirúrgico com objetivo de retirar as mamas, em particular em mulheres que estão vivendo a fase da juventude, pois, leva a transformações na sua imagem corporal, autoestima e identidade, possibilitando transparecer na manifestação de sua sexualidade, além de provocar sintomas de depressão e ansiedade.

Um dos objetivos da mastectomia é a retira total do seio infecionado pela CM, essa forma de tratamento de erradicação promove muitos sofrimentos a mulher. Por conta disso, a mastectomia provoca impacto na vida das mulheres com o CM, e isso vai modificando com toda a sua estrutura emocional e física, precisando ocorrer uma intervenção mais ativa dos profissionais e familiares com acolhimento incentivador (Silva, et al., 2020).

A extração do tecido mamário protege a pele e o mamilo, deixando os mesmos intactos, conhecido como mastectomia a denomastectomia ou subcutânea. Esse recurso terapêutico realiza a retirada total da mama. A mastectomia simples apresenta a remoção total da mama sem ocorrer a análise axilar, por ser uma cirurgia que vem com o tabu de mutilação, provocando impactos profundados no psicológico da paciente que passa por esse procedimento (Nanis, 2016).

\subsection{Desafios da Mulher Durante a Mastectomia}

No trabalho de Barbosa, et al. (2017), após equilíbrio pela variável mão funcional e tempo de cirurgia, as variáveis IMC, reconstrução mamária, expressão de peso no braço, redução do movimento (ADM) do ombro e sensação de blusa apertada demonstram-se nas QVRS. Conforme Paiva, et al. (2016), a sistematização do cuidado pode possibilitar uma assistência humanizada e individual à mulher com neoplasia mamária na sua vivência o processo cirúrgico.

Segundo Araújo, et al. (2020), diante dos muitos desafios enfrentados pelas mulheres, posteriormente a cirurgia de mastectomia, sofrimentos e tristezas invadem o psicológico, chegando ao mal-estar, em virtude do que representa a mama em sua vida. A mastectomia possui sólido impacto na feminilidade, um caráter mutilador e tange acerca da autoimagem, incentivando a mulher a vivenciar diversas implicações emocionais, físicos e sociais relacionadas à imagem corporal.

A retirada da mama, logo, provoca repercussão de repulsa para a mulher, contudo, no que se associa à sua autoimagem, provocando comportamento de isolamento ocasionado à tristeza pela mutilação, medo de rejeição, constrangimento por parte da família, amigos, dentre outros, promovendo assim, o começo de novos relacionamentos (Pereira, et al., 2016).

Para Mattias, et al. (2018), as mulheres já esperavam o diagnóstico de câncer, logo, mesmo afirmando estarem preparadas para tal resultado, diante da notícia se sentem aflitas, receberam apoio da família e ainda buscam apoio religioso para lidar com o difícil momento do diagnóstico do câncer de mama.

Para Varela, et al. (2017), é importante a compressão dos familiares começando pelos esposos e companheiros, em 
que muitas situações não estão preparadas para enfrentar o processo de adoecer e tratar o CM juntamente à esposa. Ocorrem sempre o comprometimento da autoestima e autoimagem dessas mulheres quando recebem o resultado, e como consequência, os efeitos provocados através do tratamento, pois, quando finaliza a mastectomia, a mulher se encontra com condições de fragilidade emocional.

Na pesquisa de Tapia, et al. (2021), a compreensão sobre a atenção por uma equipe interdisciplinar, é importante no cuidado de mulheres mastectomizadas, pois, o câncer e seu tratamento levam a diversas transformações na vida das mulheres, a curto e longo prazo. Lôbo, et al. (2014), consideram que as mulheres com câncer de mama apresentaram mudanças emocionais, financeiras, de satisfação sexual e nas perspectivas futuras, logo, os sintomas mais identificados eram: fadiga, insônia e perda de apetite.

De acordo com Oliveira, et al. (2017), no contexto da sexualidade confrontou mulheres afetadas pela mastectomia, como: impacto psicológico e social, devido ao tratamento cirúrgico, ou seja, transformações positivas e negativas que percorrem a terapia em mulheres com CM impacto no que se refere aos receios e tabus sobre a doença.

\subsection{Atuação do Enfermeiro na Recuperação da Paciente Mastectomizada}

Na pesquisa de Azevedo, et al. (2016), a multidisciplinaridade e a assistência de enfermagem, foram discutidas direcionadas principalmente para a atenção à saúde humanizada e qualidade para pacientes portadores de câncer de mama. Segundo Toillier, et al. (2018), afirmam que as entrevistas sempre são momentos intensos de compreensões e lembranças, onde as participantes puderam partilhar suas vivências, sensações e percepções acerca das implicações do câncer de mama em suas vidas.

De acordo com Toneti, et al. (2019) a equipe de Enfermagem é a que mais pode oferecer informações acerca dos benefícios e realizados do uso de terapias complementares ao tratamento oncológico, dado que, essa equipe possui contato direto e mais longo com o paciente no período do procedimento e reabilitação da doença, o que oportuniza ofertar e prestar os cuidados centrados no paciente e suas necessidades.

É considerável que o cuidado de enfermagem ao paciente oncológico vem demandando cada vez mais técnicas e conhecimentos que sustentem a intervenção clínica. Com isso, sendo as terapias alternativas reconhecidas pelo Conselho Federal de Enfermagem na Resolução n. ${ }^{\circ}$ 0500/2015, como especialidade ou qualificação do enfermeiro, diante da conclusão e aprovação em cursos na área em instituições de ensino reconhecidas (Brasil, 2018).

O Enfermeiro sempre está em busca de garantir condutas para a melhoria de vida dos seus pacientes, viabilizando cuidados para um bom desenvolvimento. Este profissional é dotado de responsabilidade para estabelecer busca permanente para a qualidade de vida dos pacientes, garantindo os valores onde a mulher se encontra, relacionados aos seus objetivos, prevenções e expectativas, orientando, assegurando e reforçando a segurança do mesmo (Cordeiro, et al., 2018).

Segundo Scofano, et al. (2020), o estudo evidenciou que as intervenções de enfermagem no plano de alta a mulheres submetidas à mastectomia foram baseadas na educação continuada e também de seus familiares. Segundo Silva, et al. (2021), observaram que os cuidados realizados pela equipe de enfermagem em mulheres no período de reabilitação pós-mastectomia, proporcionam grandes benefícios, quando utilizados em conjunto a ações educativas, orientação e escuta ativa.

Segundo Fernandes, et al. (2018), os profissionais sempre estão em busca de entender as necessidades de modo individualizado de cada mulher, esclarecer suas colocações, se preocupar com a forma apropriada de se referir a mulher relacionado a sua intimidade. Frequentemente o ouvir e responder é algo importante para a estabilidade clínica da paciente, são proporções satisfatórias para a maioria delas se sentirem acolhidas em suas necessidades, afirmando ainda a importância do papel do cônjuge afetivo-sexual como suporte para a reintegração da mulher mastectomizada.

Afirma Soccol, Canabarro e Pohlmann (2016), o enfermeiro tem uma função importante para a auxiliar a mulher, 
nesse processo difícil e transformador que é a extirpação da mama, que afeta de modo negativo a autoimagem da mesma. Em momentos como esse, é que as mulheres precisam do cuidado da família e do profissional de saúde para contribuir com a aceitação e contínua do recurso terapêutico.

Para Santana, Viana e Souza (2018), durante a cirurgia, a mulher precisa de ajuda emocional, para entender, aceitar e adaptar a autoimagem. Em todas as etapas da cirurgia de mastectomia, o enfermeiro deve garantir uma assistência fundamentada em evidência científica, oferecendo o melhor procedimento conhecido no momento de reduzir o sofrimento relacionado ao câncer de mama e seus possíveis prognósticos.

\section{Considerações Finais}

Observa-se a prevalência de ansiedade e depressão, que se manifesta em diversas mulheres em tratamento do câncer de mama. $\mathrm{O}$ câncer de mama se caracteriza como uma neoplasia que compromete uma das mamas, geralmente as axilas, se apresenta como um nódulo resistente que pode ser indolor ou não, manifestado através das alterações na pele, secreção mamilar ou intensa dor na mama.

Existe um crescimento contínuo de índices de neoplasia mamária, porém, cada vez mais surgem novas formas para diagnosticá-lo, assim, como alguns exames e tratamentos que geralmente não estão acessíveis à todas as mulheres, métodos que auxiliam as pacientes a detectarem o tumor, ainda de forma precoce.

Um dos tratamentos do CM, identificado é a mastectomia, que se baseia na retirada total da mama, com ou sem exclusão de linfonodos axilares. Neste contexto, que a equipe de enfermagem oferta de informações acerca dos benefícios a partir do uso das terapias complementares ao tratamento oncológico, possibilitando prestar os cuidados centrados no paciente e em suas necessidades.

Grande parte dos artigos evidenciou que a intervenção do profissional de enfermagem ocorre devido mulheres com câncer de mama apresentarem mudanças emocionais e financeiras, logo, os sintomas mais identificados são: fadiga, insônia e perda de apetite. Devido à distribuição de informações sobre este estudo sobre depressão e ansiedade em mulheres diagnosticadas com câncer de mama, no contexto pré-operatório, recomenda-se mais estudos voltados para a técnica de trabalho nesse campo específico.

\section{Referências}

Alves, E. V. (2016). O Câncer de mama e suas implicações biopsicossociais: Um estudo Bibliográfico. Monografia de Psicologia]. Boa Vista: Universidade Federal de Roraima, UFRR.

Arab, C., Demonico, B. B., Correia, C. K., Vilarino, G. T., \& Andrade, A. (2016). Câncer de mama e reações emocionais: revisão sistemática. Revista Baiana de Saúde Pública, 40(4).

Azevedo, J. J., Bezerra, K. P., Morais, F. R. R., Fernandes, A. C., Oliveira, K. S. M., \& Queiroz, J. C. (2016). As Transformações Biopsicossociais em mulheres mastectomizadas. Rev Enferm UFPE On Line. Pernambuco. Jan, 10(1), 263-72.

Barbosa, P. A., Cesca, R. G., Pacífico, T. E. D., \& Leite, I. C. G. (2017). Qualidade de vida em mulheres com câncer de mama pós-intervenção cirúrgica em uma cidade da zona da mata de Minas Gerais, Brasil. Revista Brasileira de Saúde Materno Infantil, 17, 385-399.

Batista, D. R. R., de Mattos, M., \& da Silva, S. F. (2015). Convivendo com o câncer: do diagnóstico ao tratamento. Revista de Enfermagem da UFSM, 5(3), 499-510.

Borba, P. N. (2018). Câncer de mama: os impactos psicológicos causados na mulher após o diagnóstico. Revista Científica Semana Acadêmica, (131), 1-35.

Brasil. Ministério da Saúde. Portaria n. 702, de 21 de março de (2018). Altera a Portaria de Consolidação nº 2/GM/MS, de 28 de setembro de 2017, para incluir novas práticas na Política Nacional de Práticas Integrativas e Complementares - PNPIC. Brasília, 2018.

Cordeiro, L. D. A. M., Nogueira, D. A., \& Gradim, C. V. C. (2018). Mulheres com neoplasia mamária em quimioterapia adjuvante: avaliação da qualidade de vida [Women with breast cancer in adjuvant chemotherapy: assessment of quality of life] [Mujeres con neoplasia mamaria en la quimioterapia adyuvante: evaluación de la calidad de vida]. Revista Enfermagem UERJ, 26, 17948.

Costa, E. S. D. (2019). Perfil de neoplasias mamárias em cadelas e gatas domiciliadas na mesorregião metropolitana de Belém, no período de 2016 a 2018. 
de Araujo, V. D. S. C., de Oliveira Pereira, R. M., de Souza, L. O., Almeida, M. G., de Almeida, L. D. S., da Silva Reis, M. H., ... \& Gomes, A. P. (2020). A perspectiva da autoimagem e sexualidade de mulheres mastectomizadas: revisão integrativa da literatura. Revista Eletrônica Acervo Saúde, (52), e3618-e3618.

dos Santos Scofano, B., de Lima, A. A., dos Reis Silva, R., Penna, L. H. G., de Andrade, K. B. S., \& Pinheiro, A. P. B. (2020). Ações/plano de alta da enfermagem í mulher submetida í mastectomia. Nursing (São Paulo), 23(263), 3736-3744.

da Silva, C. H. H. C., de Araújo Cunha, T. A., Dantas, C. S., \& de Jesus, C. S. (2021). A importância da enfermagem no pós-operatório de mulheres mastectomizadas com dissecção de linfonodos axilares: revisão integrativa. Research, Society and Development, 10 (6), e57210616177-e57210616177.

Fernandes, D. T., Manhães, M. V., de OB Sousa, V. C., \& dos Santos, C. M. (2018). Mulheres mastectomizadas em vigência de quimioterapia adjuvante: assistência do enfermeiro. Biológicas \& Saúde, 8(26).

Ferreira, A. S., Bicalho, B. P., Neves, L. F. G., Menezes, M. T., Silva, T. A., Faier, T. A., \& Machado, R. M. (2016). Prevalência de ansiedade e depressão em pacientes oncológicos e identificação de variáveis predisponentes. Revista Brasileira de Cancerologia, 62(4), 321-328.

Guedes, T. S. R., de Oliveira, N. P. D., Holanda, A. M., Reis, M. A., da Silva, C. P., e Silva, B. L. R., \& de Souza, D. L. B. (2018). Imagem corporal de mulheres submetidas a tratamento de câncer de mama. Jornal do Pacífico Asiático de prevenção do câncer: APJCP, 19 (6), 1487.

Instituto Nacional de Câncer José Alencar Gomes da Silva, (2019). Estimativa 2020: incidência de câncer no Brasil / Instituto Nacional de Câncer José Alencar Gomes da Silva. - Rio de Janeiro: INCA, 2019.

Lôbo, S. A., Fernandes, A. F. C., Almeida, P. C. D., Carvalho, C. M. D. L., \& Sawada, N. O. (2014). Qualidade de vida em mulheres com neoplasias de mama em quimioterapia. Acta Paulista de Enfermagem, 27, 554-559.

Mattias, S. R., de Morais Lima, N., Santos, I. D. L., Pinto, K. R. T. F., Bernardy, C. C. F., \& Sodré, T. M. (2018). Câncer de mama: sentimentos e percepções das mulheres diante do diagnóstico. Revista de Pesquisa, Cuidado é Fundamental Online, 10(2), 385-390.

Medeiros, M. B. D., Silva, R. M. C. R. A., Pereira, E. R., Melo, S. H. D. S., Joaquim, F. L., Santos, B. M. D., \& Goés, T. R. P. (2019). Percepção de mulheres com câncer de mama em quimioterapia: uma análise abrangente. Revista Brasileira de Enfermagem, 72, 103-110.

Nanis, J. S. S. (2016). Vivencias e sentimentos acerca da reconstrução mamária na qualidade de vida de mulheres submetidas a mastectomia: uma revisão integrativa.

Ohl, I. C. B., Ohl, R. I. B., Chavaglia, S. R. R., \& Goldman, R. E. (2016). Ações públicas para o controle do câncer de mama no Brasil: revisão integrativa. Revista Brasileira de Enfermagem, 69, 793-803.

Oliveira, F. B. M., Silva, F. S., \& Prazeres, A. D. S. B. D. (2017). Impacto do câncer de mama e da mastectomia na sexualidade feminina. Rev. enferm. UFPE on line, 2533-2540.

Paiva, A. D. C. P. C., Arreguy-Senna, C., da Silva Alves, M., \& de Oliveira Salimena, A. M. (2016). Construção de instrumentos para o cuidado sistematizado da enfermagem: mulheres em processo cirúrgico de mastectomia. Revista de Enfermagem do Centro-Oeste Mineiro, 6(2).

Pereira, G. B., Gomes, A. M. S. M., \& de Oliveira, R. R. (2017). Impacto do tratamento do câncer de mama na autoimagem e nos relacionamentos afetivos de mulheres mastectomizadas. Life Style, 4(1), 99-119.

Pereira, D., \& Braga, A. A. M. (2016). A mastectomia e a ressignificação do corpo no feminino. Revista Psicologia, Diversidade e Saúde, 5(1).

Santos, T. A., \& Gonzaga, M. F. N. (2018). Fisiopatologia do câncer de mama e os fatores relacionados. Rev Saúde Foco, 10, $359-366$.

Silva, E. L. S., \& Viana, E. R. (2015). A importância do diagnóstico precoce do câncer de mama e sua magnitude no universo feminino. Biológicas \& Saúde, 5(18)

Silva, M. G. D., \& Tavares, V. D. S. (2020). Qualidade de vida em pacientes mastectomizadas.

Soccol, K. L. S., Canabarro, J. L., \& Pohlmann, S. C. (2016). Atuação da enfermagem frente a mulher com câncer de mama: revisão de literatura. Multiciência online. [Internet], 2(4), 71-88.

Steffens, S. R. (2018). Sexualidade E Câncer De Mama. Anuário Pesquisa e Extensão Unoesc São Miguel do Oeste, 3, e19671-e19671.

Teixeira, L. A., \& Araújo, L. A. (2020). Câncer de mama no Brasil: medicina e saúde pública no século XX. Saúde e Sociedade, 29.

Toneti, B. F., Avelar, J. M. D. P., Sousa, F. H., Toneti, A. N., Sonobe, H. M., \& Sawada, N. O., (2019). O significado de uma terapia integrativa de relaxamento guiado para mulheres com câncer de mama. Revista da Escola de Enfermagem da USP, 53.

Viana, D. D. A., de Santana, C. C. C., \& Souza, J. R. S. (2018). Análise das ações de enfermagem nas fases cirúrgicas da mastectomia. Revista Brasileira de Inovação Tecnológica em Saúde-ISSN: 2236-1103.

Varela, A. I. S., da Rosa, L. M., Sebold, N., Laverde, A. G., Maçaneiro, A., \& Erdmann, A. L. (2017). Comprometimento da sexualidade de mulheres com câncer de mama. Enfermagem em Foco, 8(1), 67-71.

Zapponi, A. L. B., Tocantins, F. R., \& Vargens, O. M. D. C. (2015). O enfermeiro na detecção precoce do câncer de mama no âmbito da atenção primária. Rev enferm UERJ, 23(1), 33-8.

Zúñiga-Tapia, R., Panobianco, M. S., Prado, M. A. S., \& Henríquez, P. C. (2021). Experiencia en centro de rehabilitación de mujeres mastectomizadas al início de la pandemia por COVID-19. Revista Gaúcha de Enfermagem, 42. 